\title{
Extraction of Sensory Parameters from a Neural Map by Primary Sensory Interneurons
}

\author{
Gwen A. Jacobs ${ }^{1}$ and Frederic E. Theunissen ${ }^{2}$ \\ ${ }^{1}$ Center for Computational Biology, Montana State University, Bozeman, Montana 59717, and 2Department of \\ Psychology, University of California, Berkeley, California 94720
}

\begin{abstract}
We examine the anatomical basis for the representation of stimulus parameters within a neural map and examine the extraction of these parameters by sensory interneurons (INs) in the cricket cercal sensory system. The extraction of air current direction by these sensory interneurons can be understood largely in terms of the anatomy of the system. There are two critical anatomical constraints. (1) The arborizations of afferents with similar directional tuning properties are located near each other within the neural map. Therefore, a continuous variation in stimulus direction causes a continuous variation in the spatial pattern of activation. (2) The restriction of the synaptic connections of an interneuron to a unique set of afferents results from the unique anatomy of that interneuron: its dendritic arbors are
\end{abstract}

located within restricted regions of the afferent map containing afferents with a limited subset of directional sensitivities. The functional organization of the set of four interneurons studied here is equivalent to a Cartesian coordinate system for computing the stimulus direction vector. For any air current stimulus direction, the firing rates of the active interneurons could be decoded as Cartesian coordinates by neurons at successive processing stages. The implications of this Cartesian coordinate system are discussed with respect to optimal coding strategies and developmental constraints on the cellular implementation of this coding scheme.

Key words: sensory maps; insect; functional neuroanatomy; sensory interneurons; spatiotemporal patterns; neural coding
In most sensory systems, neurons form a map of sensory space through the organized projection pattern of their axon terminals. One consequence of this anatomical organization is that the ensemble response of a population of neurons to a particular sensory stimulus will be represented as a unique spatiotemporal pattern of activity within that region of the brain. To understand how these spatiotemporal patterns of activity emerge from the ensemble activity, and how the information contained in the patterns is accessed and encoded by higher levels of the nervous system, the functional architecture of the constituent neurons must be determined. This is an exceedingly difficult task in most sensory systems. However, in the cricket cercal system, it has been possible to collect adequate anatomical and physiological data about individual neurons in sufficient numbers to create a comprehensive database representing the functional organization of one entire processing stage of this system (Troyer et al., 1994).

We have used this database to address two specific questions related to the computation and transfer of stimulus parameters between layers of neurons within a sensory system. (1) What is the algorithm for transmitting information encoded by one population of neurons to another? (2) How is the algorithm implemented within the network of neurons, and what are the structural constraints on that implementation?

The primary afferent input to the cricket cercal sensory system comes from an ensemble of mechanosensory afferents that inner-

\footnotetext{
Received Sept. 29, 1999; revised Jan. 27, 2000; accepted Feb. 7, 2000.

This work was supported by grants from the National Institute for Mental Health (RO158525) and the National Science Foundation (IBN 9421185). We thank John Miller for valuable discussions, and Sandy Pittendrigh, Ben Livingood, and Alex Dimitrov for technical assistance.

Correspondence should be addressed to Dr. Gwen A. Jacobs, Center for Computational Biology, 30 AJM Johnson Hall, Montana State University, Bozeman, Montana 59717-3505. E-mail: gwen@nervana.montana.edu.

Copyright (C) 2000 Society for Neuroscience $0270-6474 / 00 / 202934-10 \$ 15.00 / 0$
}

vate filiform hairs on two sensory appendages, called cerci, located at the rear of the animal's abdomen (see Fig. $1 A$ ). Filiform hairs are displaced by air motion and constrained by a cuticular socket to move in a single plane with respect to the cercal surface. The mechanoreceptors are sensitive to both the direction and the dynamics (frequency, velocity) of air currents (Shimozawa and Kanou, 1984a,b; Landolfa and Miller, 1995; Roddey and Jacobs, 1996). The mechanoreceptors project their axons into the CNS and provide direct excitatory input to an ensemble of sensory interneurons (INs) (see Fig. 1B) (Bacon and Murphey, 1984). These afferents and interneurons are sensitive to the direction and dynamics of air currents (see Fig. 1C,D) (Theunissen et al., 1996).

The mechanosensory afferents arborize in specific locations in the CNS according to their directional tuning properties (Bacon and Murphey, 1984). Afferents tuned to similar air current directions overlap extensively in the neuropil, whereas those tuned to opposite directions are spatially segregated. This organized projection pattern creates a continuous map of air current direction in the CNS (see Fig. 2) (Jacobs and Theunissen, 1996; Paydar et al., 1999). There are two hemimaps of air current direction in the terminal abdominal ganglion, each one formed by afferents from each cercus. Each map contains a complete representation of air current direction, and the two maps are mirror images of one another. The structure of this map and the structure of the interneurons imbedded within this map are the primary determinants of the functional sensitivity of those interneurons.

\section{MATERIALS AND METHODS}

Histological staining techniques

Adult female crickets were used within $24 \mathrm{hr}$ of their imaginal molt (Bassetts Cricket Ranch, Visalia, CA). Each individual afferent was stained with cobalt chloride and silver-intensified using methods developed by Bacon and Altman (1977) that were further modified by Johnson 
and Murphey (1985) and Jacobs and Nevin (1991). The interneurons were stained with cobalt chloride and developed using the same methods.

\section{Computer reconstruction of stained neurons}

A computer-controlled digitizing light microscope was used as the dataentry device. Tissue containing a single dye-filled nerve cell was mounted on the microscope stage, and the operator controlled the precise movement of the neuron in three dimensions by means of three high-precision motors, each mounted on a different axis of the mechanical microscope stage. The neuron was moved under the microscope so that its branches were traced under a video cursor, superimposed over a frame-grabbed video image of the tissue. Movements of the microscope stage were monitored by linear encoders mounted to the stage, each encoder having a resolution of $0.2 \mu \mathrm{m}$. A set of $x, y$, and $z$ coordinates was recorded for the endpoints of each dendritic segment, along with the mean diameter of the segment between the endpoints. These microscopy and computer techniques have been described in detail elsewhere (Jacobs and Nevin, 1991).

\section{Database of identified neurons}

All of the reconstructed afferents and interneurons were scaled and aligned to a common coordinate system and entered into a database using a suite of software programs called NeuroSys that was developed in our laboratory (http://www.nervana.montana.edu/NeuroSys). The data currently in the database consist of more than 200 primary afferents and several identified primary sensory interneurons.

\section{Estimating the total surface area of the varicosity membrane of an afferent}

Three to five examples of each type of identified afferent were selected from the database. The terminal varicosities of each cell were transformed into a continuous function representing the anatomical location and distribution of the varicosity membrane surface area. Specifically, we calculated the varicosity membrane surface area per unit volume of neuropil, which is equivalent to the surface area density. The density functions for each set of three to five afferents were averaged into a mean density function for that afferent type. These density functions were visualized as three-dimensional (3-D) space-filling clouds of points. Details of the statistical methods used for this procedure are described by Jacobs and Theunissen (1996).

\section{Ensemble map of the entire afferent projection}

The ensemble density function of the entire set of afferents was determined by calculating the linear sum of all individual density functions described above. To do so, the space corresponding to the cercal glomerulus was divided into cubical voxels that were $7 \mu \mathrm{m}$ on each side, and the net density was calculated for each voxel. Because there was a substantial degree of overlap between the density functions of afferents having similar directional sensitivities (Jacobs and Theunissen, 1996), the net density function in many voxels corresponded to synaptic varicosity surface area from afferents having different directional sensitivities. The mean directional sensitivity of each voxel was calculated by computing the vector sum of the peak directional sensitivities of all afferents projecting into that voxel, weighted by the relative densities of those afferents. For visualization, each voxel was displayed as a population of dots, with the relative density of the synaptic membrane coded by the density of the dots and the mean directional sensitivity coded with color.

\section{Predictions of steady-state activity patterns from the database}

Presentation of a unidirectional air current stimulus to a cricket changes the activity of each cercal filiform afferent: its activity either increases above or decreases below its baseline activity level. The change in activity is proportional to the cosine of the angle between the stimulus direction and the peak sensitivity direction of the afferent (Landolfa and Miller, 1995). We derived predictions of the ensemble activity pattern that would emerge from stimulation of the entire ensemble of afferents from the database. To do so, we calculated the contribution of each individual afferent to each voxel in the ensemble map by scaling its predicted physiological response by its varicosity density within that voxel. The physiological response of the afferent was calculated as the cosine of the difference in angle between the peak directional tuning of the afferent and the angle of the stimulus with respect to the animal's body. The response of the entire system of afferents was then calculated as the sum of responses from all afferents in the database at each voxel. The predicted response patterns were imaged as changes in the levels of activity in the neural map relative to the baseline level of activity. A gray scale was used to indicate relative levels of activity, with white indicating maximum activity and black indicating minimum activity. The baseline activity level in the afferents was represented as midgray (see Fig. 3, inset). In this manner, images could be generated to predict the relative response levels throughout the map for any given stimulus.

\section{Predictions of connectivity relationships between neurons}

For this analysis, we were interested in the varicosity surface density near the dendritic arborizations of the chosen interneuron. A method was derived to calculate the effective overlap between the membrane of the interneuron dendrites and the afferent terminal varicosities. Note that this operation is equivalent to multiplying a spatial filter (in the shape of an interneuron) with the structure of the afferent map. To do so, the local afferent varicosity surface density was computed at all points along the dendritic arbor of the digitized interneuron, excluding branches $>2 \mu \mathrm{m}$ in diameter. The net directional tuning of these afferents was then calculated, and the dendrites of the interneuron were color-coded according to the net directional tuning.

\section{Predictions of relative levels of activity in interneurons}

To predict the relative levels of afferent input onto an interneuron in response to a specific sensory stimulus, the "image" of the stimulusevoked response across the entire afferent ensemble was masked onto the dendritic structure of the interneuron as follows. The spatial pattern of activity within the afferent population in response to a stimulus was first predicted. Next, this pattern of afferent activity was mapped onto the dendritic structure of the interneuron, as described in the preceding section. Each of the dendritic segments of the interneuron was assigned a gray scale value corresponding to the activation level of the afferent terminals in the voxels overlapping with that dendritic segment. These predictions represent the level of excitatory input to each dendritic region of the interneuron, relative to the baseline level of activation that segment would get in the absence of any stimulation. A dendritic segment with increased activation over baseline would appear lighter than the baseline gray level, and a segment with decreased activation would appear darker than the baseline gray level.

\section{Directional tuning curves}

Several figures show directional tuning curves for afferents and/or interneurons. In this study, only the shapes of the tuning curves are relevant and not their absolute amplitudes. All tuning curves shown in these figures were derived from experimental measurements published earlier, as follows.

Afferents. All afferent directional tuning curves presented in this report were derived from averaging the directional tuning curves measured from 60 receptors, as described in Landolfa and Miller (1995). For each receptor, a unidirectional air current was presented eight times at each of 16 different directions around the animal's body in the horizontal plane. The responses were recorded at each stimulus direction and quantified as the number of spikes generated in response to the stimulus. The directional tuning curve of each afferent was plotted as the fraction of its maximum response (i.e., the response elicited at the direction of its peak sensitivity) versus stimulus direction. Thus, all tuning curves were scaled to the same maximum value of 1.0, regardless of the actual number of spikes elicited at their peak response directions. The tuning curves from the 60 afferents were then shifted with respect to their actual peak sensitivity directions, so as to align their peaks. The curves were then averaged. This average curve resembles a cosine function, with slight deviations from the pure cosine at the peak and trough. Note that these afferents, all associated with "long" filiform hairs ( $\sim 1 \mathrm{~mm}$ in length), fire spontaneously in the absence of any stimulus. Therefore, the response at each direction is either an elevation above baseline or depression below baseline; this baseline activity is indicated by the thick horizontal line through the center of the curves.

Interneurons. All interneuron directional tuning curves presented in this report were derived from averaging the directional tuning curves measured from 18 interneurons of this type, as described elsewhere (Miller et al., 1991.) For each interneuron, a unidirectional air current was presented eight times at each of 16 different directions around the animal's body in the horizontal plane. The responses were recorded at each stimulus direction and quantified as the number of spikes generated in response to the stimulus. The directional tuning curve of each inter- 
Figure 1. Functional organization of the cricket cercal sensory system. $A$, Schematic diagram of the common house cricket, Acheta domestica, showing the location of the abdominal nerve cord. The cerci are two abdominal appendages projecting from the rear of the animal's body. Both cerci are covered with mechanosensory hairs, each of which is innervated with a single sensory neuron. The axons of the sensory neurons project into the terminal abdominal ganglion, located at the caudal end of the abdominal nerve cord. $B$, An image from the database, showing the outline of the terminal abdominal ganglion and segments of four different reconstructed neurons. Three of the neurons are primary sensory afferents, whose axons can be seen entering the terminal ganglion through the cercal nerves. The fourth neuron is a primary sensory interneuron, the axon of which projects anterior to higher centers of the nervous system. Display of its cell body has been suppressed to prevent obscuration of the dendrites. $C$, Directional tuning curves of the three primary sensory afferents shown in $B$, plotted as relative response amplitude versus stimulus direction. The center horizontal line indicates baseline activity level. Stimuli directed at the front of the animal's body is indicated by $0^{\circ}$, and angles increase clockwise. The response curves are approximately sinusoidal and were derived from physiological measurements described in Materials and Methods. $D$, Directional tuning curve of the primary sensory interneuron shown in $B$, plotted as relative response amplitude versus stimulus direction. Conventions are as in $C$. This tuning curve was derived from physiological measurements described in Materials and Methods and is approximated here by a truncated cosine curve fit to the actual stimulus-response data.

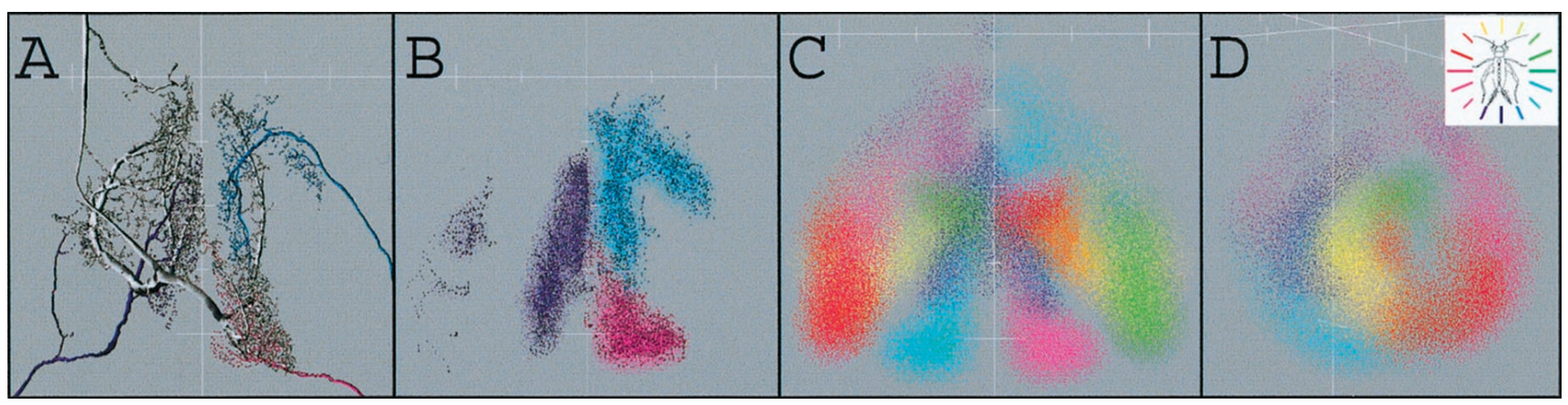

Figure 2. Mapping of stimulus direction in the cricket cercal system. A, Spatial relationships between three primary afferents and one Right 10-2 (R10-2) sensory interneuron. Primary afferents have been color-coded according to the peak of their directional tuning, indicated by the color wheel in $D$, inset. $B$, Relative positions of the terminal arbors of three primary afferents within the terminal abdominal ganglion. Afferents have been color-coded according to their peak directional tuning, according to the color wheel in $D$, inset. Each colored cloud represents an average density function of the membrane surface area of the synaptic varicosities of one afferent, calculated from five examples of the same identified afferent. $C$, Combined image of the entire ensemble of sensory afferents that innervate the long filiform mechanoreceptor hairs on both cerci. This represents a dorsal view of the terminal abdominal ganglion. The color of each cloud in the ensemble indicates the peak sensitivity direction of the corresponding afferent. Afferents from each cercus form a hemimap on one side of the ganglion. The two hemimaps are mirror images of each other across the midline of the ganglion. $D$, Map of air current direction, as in $C$, viewed from the ventral lateral aspect of the ganglion. Inset, Color wheel indicating the color coding for stimulus direction with respect to the cricket's body. In all figures, the distance between ticks on the cross hairs is $40 \mu \mathrm{m}$.

neuron was plotted as the fraction of its maximum response (i.e., the response elicited at the direction of its peak sensitivity) versus stimulus direction. Thus, all tuning curves were scaled to the same maximum value of 1.0, regardless of the actual number of spikes elicited at their peak response directions. The curves were then averaged. The distributions of the individual responses around this mean curve were shown to be extremely small, indicating that the mean curve was a statistically acceptable representation of the individual cells' directional tuning curves. The equation for a truncated cosine function was then fit to these data points and found to fit the data as well as the mean curve. For the studies reported here, we use this best-fit truncated cosine to represent the mean directional tuning curves of the interneurons.

\section{RESULTS}

\section{Representation of sensory stimuli within the neural map}

On each cricket cercus, there are $\sim 1000$ cercal filiform mechanosensory hairs, each of which is innervated by a single mechanosensory receptor neuron. The primary afferents that innervate the long $(\sim 1 \mathrm{~mm})$ filiform hairs fire action potentials spontaneously in the absence of air current stimuli. Each afferent responds to unidirectional air currents with either an increase or a decrease in its firing rate, relative to its baseline rate. The response amplitude depends on stimulus direction: each afferent has a direction of peak sensitivity, and stimuli from other directions elicit responses that decrease in amplitude by an amount proportional to the cosine between the stimulus direction and the direction of peak sensitivity of the afferent (Figs. 1, 2) (Landolfa and Miller, 1995).

The different afferents innervating mechanosensory hairs on each cercus have a wide range of peak tuning directions that span $360^{\circ}$ in the horizontal plane. Any air current stimulus in the horizontal plane will deflect all of the mechanosensory hairs on each of the two cerci to some extent, depending on the axes of movement of the hairs. As a direct consequence, activity levels in some afferents will increase, whereas the activity in others will decrease. Because the sensory afferents project into the cercal glomerulus to form a continuous spatial map of stimulus direction, then these differential afferent firing patterns elicited by 

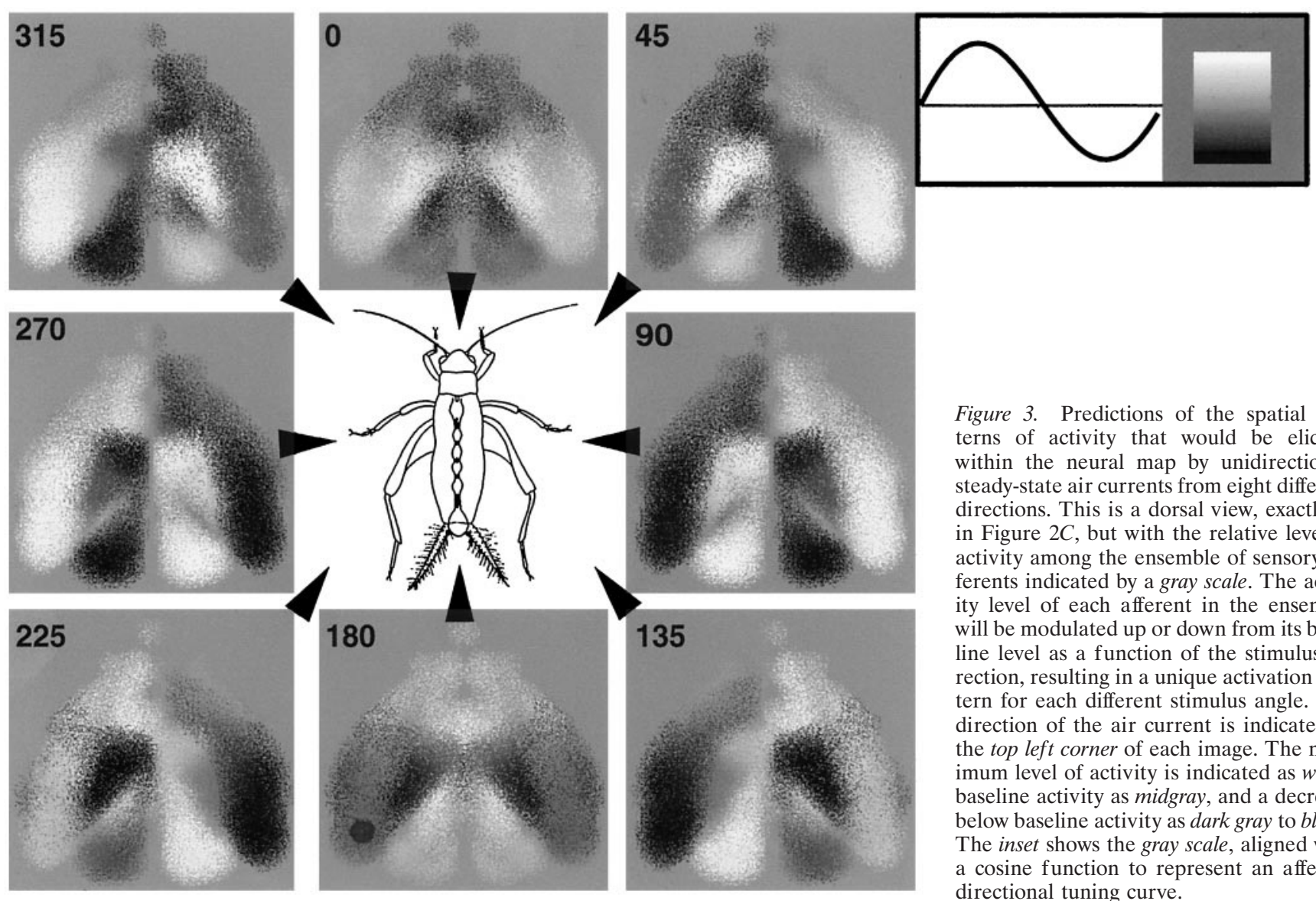

Figure 3. Predictions of the spatial patterns of activity that would be elicited within the neural map by unidirectional, steady-state air currents from eight different directions. This is a dorsal view, exactly as in Figure 2C, but with the relative level of activity among the ensemble of sensory afferents indicated by a gray scale. The activity level of each afferent in the ensemble will be modulated up or down from its baseline level as a function of the stimulus direction, resulting in a unique activation pattern for each different stimulus angle. The direction of the air current is indicated in the top left corner of each image. The maximum level of activity is indicated as white, baseline activity as midgray, and a decrease below baseline activity as dark gray to black. The inset shows the gray scale, aligned with a cosine function to represent an afferent directional tuning curve.

stimuli originating from different directions will result in different ensemble spatial patterns of activity within the cercal glomerulus.

Figure 3 shows the spatial patterns of activity that we predicted in the entire ensemble of long-hair afferents in the database, for unidirectional air current stimuli originating from eight different directions. The relative level of activity at each point in the map is shown in gray scale. The background gray corresponds to the spontaneous activity level of the afferents (i.e., in the absence of any stimulus, there would be no visible pattern.) Lighter grays indicate a net excitation of afferents above the baseline, and darker grays represent a net suppression of activity below the baseline. Maximal excitation of an afferent by an air current directed along its peak tuning axis would cause its contribution to the overall pattern to be indicated as white. A stimulus from $90^{\circ}$ away from its peak tuning direction would set its tone to midgray; a stimulus from the anti-preferred $\left(180^{\circ}\right)$ direction would set its tone to black. Each afferent responds to the stimulus according to its specific directional tuning curve.

A unique pattern of activity is generated within the population of afferents for each stimulus direction. Note that each of these patterns is actually a three-dimensional pattern but is presented here as a projection in two dimensions. The patterns vary continuously as a function of the stimulus direction. Stimuli that are similar in direction elicit activity in similar regions of the map, whereas stimuli from opposite directions are represented by patterns that are tonal inverses of one another. Each spatial pattern has approximately the same net amount of excitatory area; it is the shape and position of the areas of peak excitation and inhibition that vary with air current direction.

\section{Computation of stimulus direction by interneurons}

The spatial patterns of activity produced by the afferents constitute the neural images of sensory stimuli that must be decoded by the network of postsynaptic neurons. Thus, it is the anatomical arrangement of the primary afferent arborization that sets up the subsequent computations of stimulus direction by the primary sensory interneurons. Previous work on this system has shown that several primary sensory interneurons in this system are directionally tuned to air currents (Bacon and Murphey, 1984; Jacobs et al., 1986; Miller et al., 1991). The tuning curves of at least four of these cells are very similar to those of the afferents and are well approximated as truncated cosine functions (Miller et al., 1991).

Figure 4 shows these four identified sensory interneurons: left (L)10-2, right (R)10-2, L10-3, and R10-3. Also shown are truncated cosine functions fit to their experimentally measured directional tuning curves, elicited in response to unidirectional air current stimuli (Miller et al., 1991). All cell types have tuning curves that are statistically indistinguishable in shape but are shifted from each other at $90^{\circ}$ intervals around the horizontal plane. Together, the tuning curves of these four interneurons span the entire range of stimulus directions in the horizontal plane.

How do these interneurons derive their directional tuning characteristics from the neural map of air current direction? Our working hypothesis is that the directional tuning properties of primary sensory interneurons can be explained primarily on the basis of the anatomical overlap between their dendritic trees and 

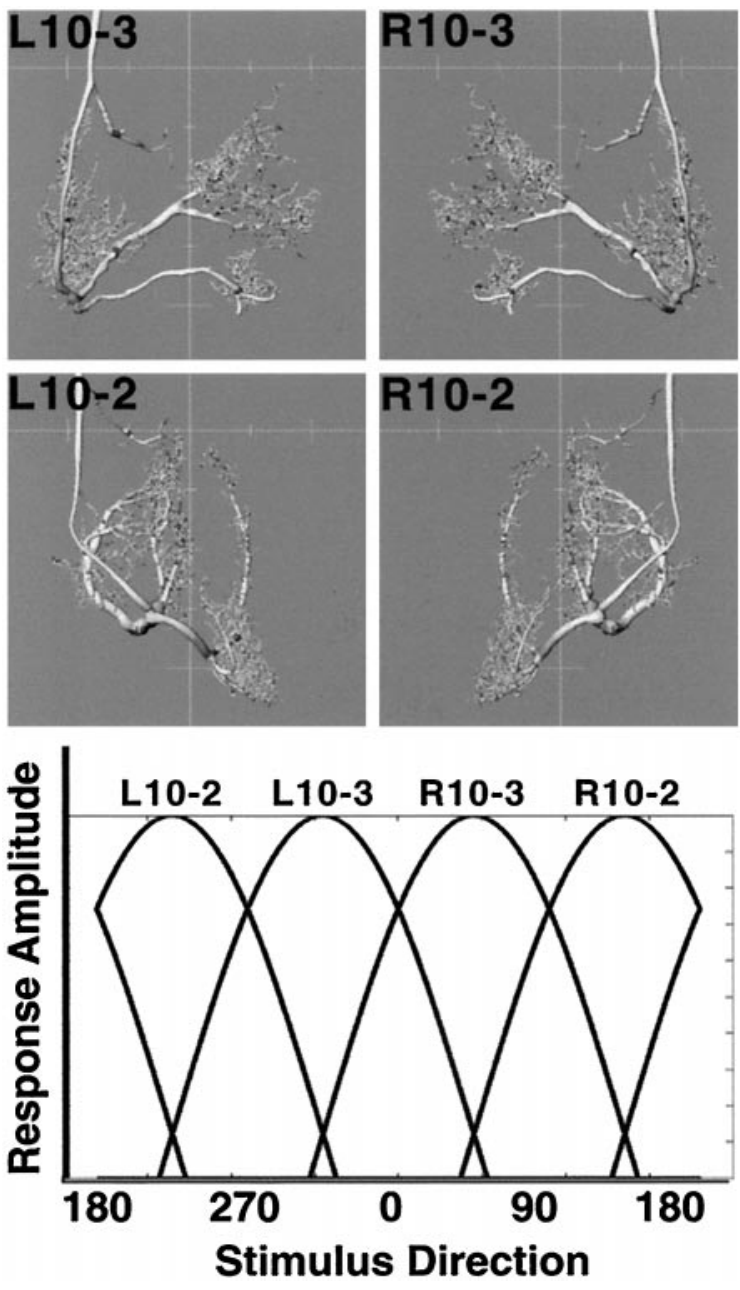

Figure 4. Anatomical reconstructions of four different primary sensory interneurons and their directional tuning curves. Top four panels are the images of L10-3, R10-3, L10-2, and R10-2. Each neuron was stained and reconstructed in $3-\mathrm{D}$, scaled, and aligned to the database. The cell bodies of the neurons have been removed so as not to obscure the dendritic branching patterns. The distance between the ticks on the horizontal and vertical axis on each image is $40 \mu \mathrm{m}$. Bottom panel, Directional tuning curves of each interneuron to unidirectional air current stimuli, plotted as relative response amplitude versus stimulus direction. These tuning curves are truncated cosine curves fit to experimentally measured stimulus-response data, as described in Materials and Methods.

the map of air current direction formed by the primary sensory afferents (Bacon and Murphey, 1984; Jacobs and Miller, 1985; Jacobs et al., 1986). Different interneurons have very different dendritic arborizations, which may allow them to receive distinct sets of excitatory inputs. We tested this hypothesis by comparing the positions of the dendrites of the identified interneurons described above with predicted patterns of activity generated within the neural map of air current direction.

Figure 5 shows the four INs and their spatial relationships to the map of air current direction in the terminal ganglion. Figure $5 A$ is a stereo pair of IN L10-3 superimposed over the afferent map. The colored clouds represent the combined ensemble of sensory afferent density clouds, color-coded for directional sensitivity identically as they were in Figure $2 C$. Note that each dendritic segment of the interneuron has been assigned a color identical to the local color of the afferent cloud. Figure $5 B$ shows this same color-coded representation of the interneuron, this time without the ensemble afferent density clouds (i.e., the dendrites of the interneuron have been used as a "mask" for the afferent density map). This illustrates the spatial location of the dendrites of the interneuron with respect to the map of stimulus direction synthesized by the afferent projections into this region of the glomerulus. This image represents a quantitative, first-order prediction of the spatial distribution of afferent inputs onto this interneuron as a function of the afferents' peak directional tuning. Figure $5 C-E$ shows similar predictions for the other three interneurons in this class.

These predictions suggest that the distribution of afferent inputs to each of the four interneurons is unique. Each of the four INs has a large dendritic arbor that extends over a broad region of the map. Despite this extensive arborization, the different subarbors of each IN sample regions of the map that are relatively similar in directional tuning. For example, in Figure $5 C$, interneuron L10-2 projects to areas of the map that are tuned to air current directions centered around $225^{\circ}$. The range of directional tuning inputs that this neuron could receive is $\sim 135-315^{\circ}$. Different interneurons sample overlapping but significantly different regions. For example, IN L10-3 overlaps with regions of the map encompassing air current directions from 45 to $225^{\circ}$, centered on $315^{\circ}$. Thus, the position of the dendrites of each of these interneurons ensures that each one has access to a broad range of excitatory inputs and that these inputs are from regions of the map that represent continuous regions of sensory space.

These relationships were quantified by calculating the amount of anatomical overlap between all the primary afferents and each of the four interneurons and plotting the amount of overlap versus the directional tuning of the afferent. Figure 6 shows these relationships for the four interneurons. Each interneuron overlaps with a broad range of afferents, yet the distribution of these inputs is unique to each IN. The largest amount of overlap correlates roughly with the peak of the directional tuning curve of each IN. As a group, these INs cover the entire stimulus range represented within the afferent population.

\section{Dendritic shapes as detectors of spatial patterns of activity}

The results presented thus far support the hypothesis that the directional tuning properties of interneurons are very closely tied to their dendritic structures. Because the input to the interneurons from the sensory afferents takes the form of a threedimensional spatial pattern, then the decoding mechanism used by the interneurons could be a "spatial filter" function: the interneuron responds maximally when the afferent activation pattern matches its dendritic architecture and responds less as the activation pattern diverges from that shape. If a pattern of activity is generated that is the inverse of the shape of the IN (i.e., suppression of activity through presentation of an anti-preferred stimulus), then activity in that IN is suppressed. Each interneuron should be sensitive to a specific range of spatial patterns that correspond to the locations of its dendrites. Because each interneuron has a different shape, each should be sensitive to only a subset of the activity patterns.

We examined this hypothesis by predicting the level of excitatory input onto the dendrites of each interneuron as a function of the spatial pattern of activity within the map for stimuli from different directions. Figure 7 illustrates our approach. The top panel of Figure 7 shows a stereo pair image of the activity pattern predicted within the afferent map in response to a stimulus at $225^{\circ}$ with respect to the animal's body. This direction is known from 

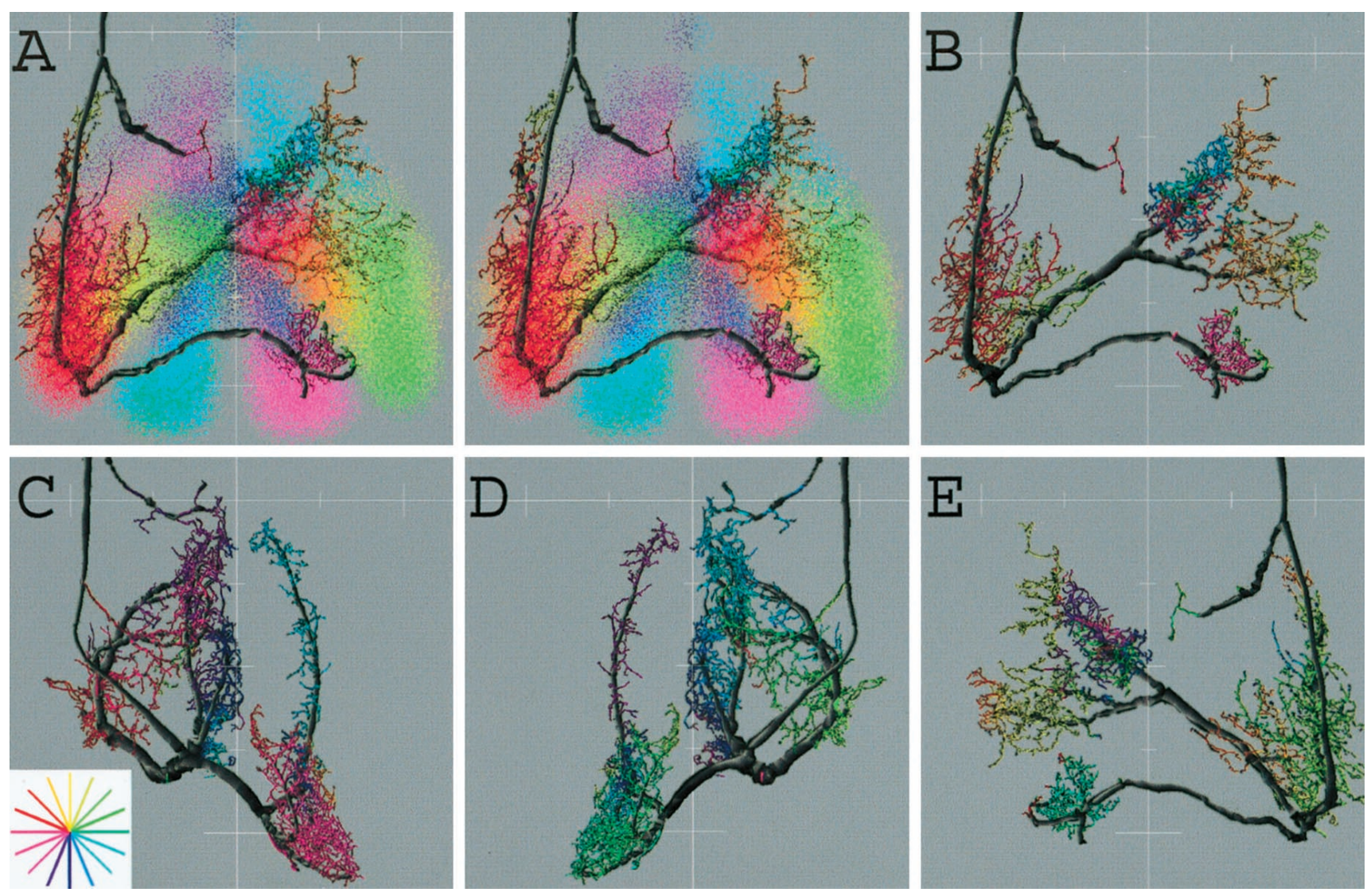

Figure 5. Prediction of the distribution of excitatory inputs to the dendrites of four identified interneurons. $A$, Stereo image of IN L10-3 in register with the map of air current direction formed by the primary sensory afferents. The dendrites of the interneuron have been color-coded according to their spatial location within the map. The color coding represents a quantitative prediction of the distribution of excitatory input to each dendritic region. $B$ shows this distribution of inputs on R10-3, but without the afferent density clouds. $C-E$ show similar predictions for the remaining three types of sensory interneurons $(C, \mathrm{R} 10-3 ; D, \mathrm{~L} 10-2 ; E, \mathrm{R} 10-2)$. Note that each interneuron may receive input from sensory afferents tuned to a wide range of directions. However, the distribution of these inputs is unique to each interneuron. The distance between ticks on the horizontal and vertical axis on each image is $40 \mu \mathrm{m}$.

earlier electrophysiological studies to be the optimal stimulus direction for IN L10-2 (Miller et al., 1991.) As in Figure 3, the relative activity levels in the map are coded with a gray scale. In the center stereo pair, the 3-D structure of IN L10-2 (shown in black) is superimposed with this afferent response pattern. Note that areas of the map that show high levels of activity (white regions) are located in the same regions as most of the dendrites of the IN. In the lower stereo pair, the dendrites of the interneuron have been used to mask the afferent activity pattern. That is, each dendritic segment of the interneuron has been assigned a tone of gray identical to the local color of the afferent activity level within the map. Here, the distribution and level of activity can be seen to vary over the different dendritic regions. Most of the dendrites appear white, which indicates that the afferent input to these dendritic regions is maximally activated by the stimulus. Other dendritic regions show lower levels of activation, as indicated by darker gray levels. Qualitatively, the spatial filter formed by the dendritic architecture of this IN is well matched to the pattern of activity that we predict would be elicited by a stimulus at the optimal direction of that IN.

Just as the spatial pattern of activity in the map changes as a function of stimulus direction, so does the level of excitatory input to the different dendritic regions of the interneuron. Figure 8 shows the predictions of the level of excitatory input to IN L10-2 for four orthogonal stimulus directions: $45,135,225$, and $315^{\circ}$.
These images are shown for qualitative comparison to the truncated cosine curve corresponding to the average directional tuning curve of this type of IN. For stimuli at $225^{\circ}$, which is the peak sensitivity direction for this IN, a large portion of the dendritic arbor is activated maximally. At the opposite (anti-preferred) direction, most of the dendritic segments are black, indicating that most of the afferent input is totally suppressed. At intermediate directions, there is some balance between excitation and suppression.

Similar functional relationships hold true for the three other types of interneurons studied here. Figure 9 shows the levels of excitation in the dendritic trees of the four interneurons, for each of four different stimulus directions. These four directions (45, 135,225 , and $315^{\circ}$ ) correspond to the peak tuning directions of the different interneurons. Note that the best match between the structure of each interneuron and the different predicted patterns of afferent activation corresponds to the peak tuning direction of that IN (IN L10-3, 45; IN R10-2, 135 ; IN L10-2, 225 ; IN R10-3, $315^{\circ}$ ). Each shows the greatest suppression at a direction opposite to its peak tuning direction.

\section{DISCUSSION}

The algorithm for the computation of stimulus direction at this primary processing stage in the cercal system is very straightforward. The afferents are broadly tuned to air current direction and 

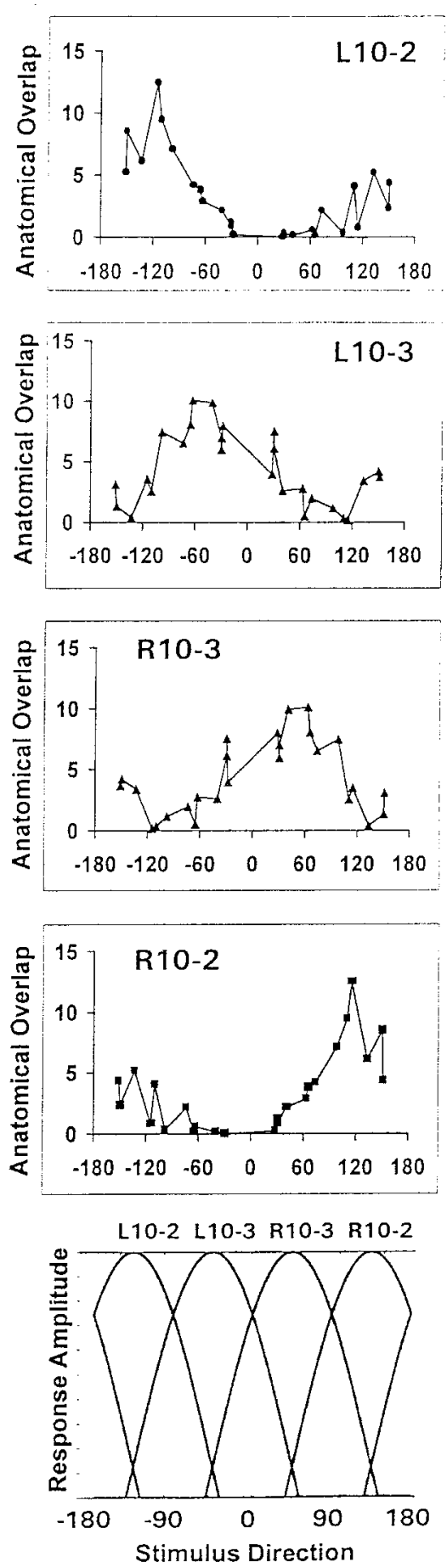

Figure 6. Extent of anatomical overlap between primary sensory interneurons and primary afferents, as a function of the peak directional sensitivity of the afferents. The top four panels show the percentage of anatomical overlap between each primary sensory interneuron and the whole range of sensory afferents from both cerci. Each interneuron overlaps differentially with the afferent ensemble and has a great deal more overlap with some afferents than with others. The bottom panel shows the truncated cosine functions that are the best-fit approximations to the directional tuning curves of the sensory interneurons, determined as described in Materials and Methods. It is clear that the directional tuning curve of each interneuron is centered at a stimulus direction that corresponds to the peak in the distribution of afferents with which it has the greatest anatomical overlap.
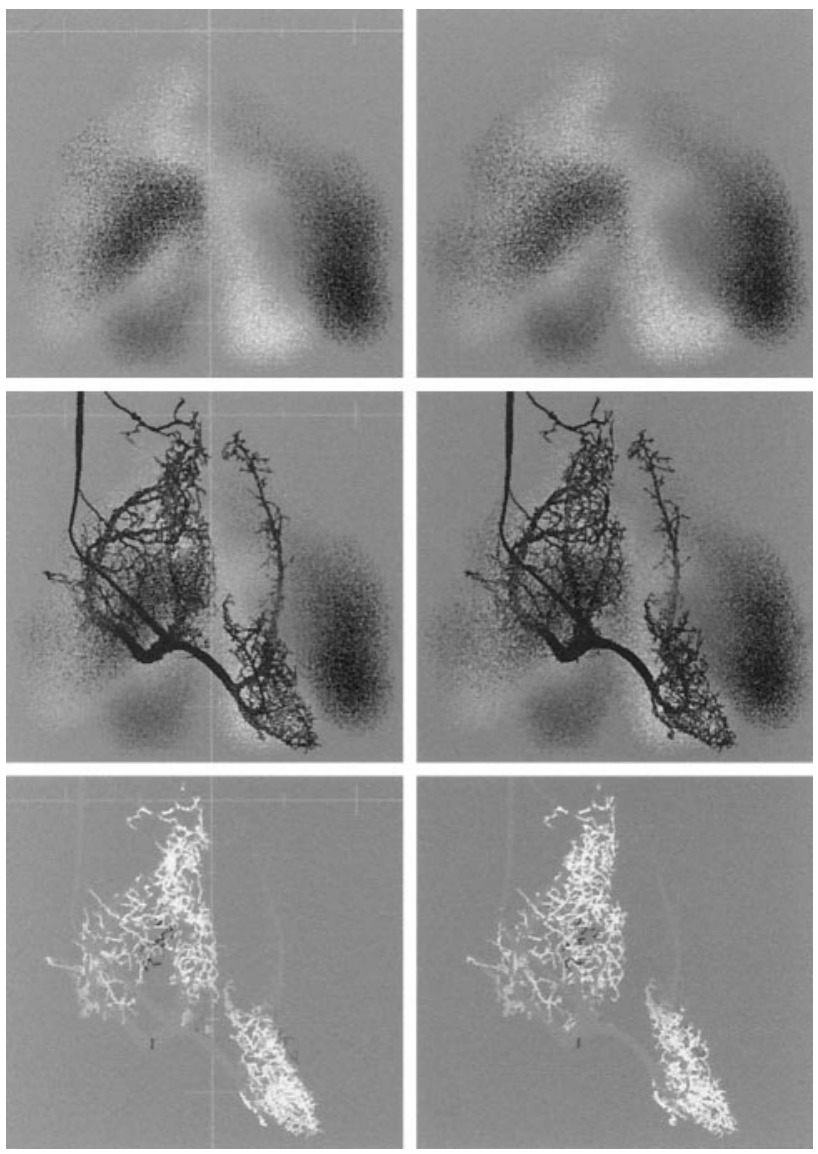

Figure 7. Prediction of the relative levels of excitatory input onto the dendrites of an interneuron, in response to a stimulus from that cell's optimal stimulus direction. Top, Stereo pair image of the predicted spatial pattern of activity within the afferent map in response to an air current directed at the animal at $225^{\circ}$. For each population of afferents tuned to a specific direction, the maximum level of activity is indicated as white and baseline activity as midgray, and a decrease below baseline activity is indicated as dark gray to black. Middle, Stereo pair image of IN L10-2 imbedded within this activation pattern. Note that the dendrites occupy regions of relatively high activity within the map (white areas). Bottom, Stereo pair image of the dendrites of the interneuron, in the absence of the activity-coded afferent density clouds. Here, the structure of the dendrites of the IN has been used to mask the afferent activity pattern. That is, each dendritic segment of the interneuron has been assigned a tone of gray identical to the local color of the afferent activity level within the map. This gray scale-coding therefore represents a first-order prediction of the relative level of excitatory input to the dendrites from afferents activated by the stimulus.

have directional response curves that are well estimated by cosine functions. These afferent tuning curves form the basis function set from which the interneuron tuning curves are derived. The stimulus-response curves of the four interneurons studied here appear to be synthesized through linear summation and subsequent thresholding of restricted subsets of these afferent tuning curves. Because the subset of afferents to which each interneuron connects have a very restricted range of peak tuning directions, then the response of each interneuron also displays directional sensitivity. The net directional sensitivity of the interneuron is the weighted (and thresholded) average of the tuning curves of the afferents that synapse onto that interneuron.

The extraction of air current direction by these sensory interneurons can be understood largely in terms of the anatomy of the system. First, the afferent projection is arranged in such a way 

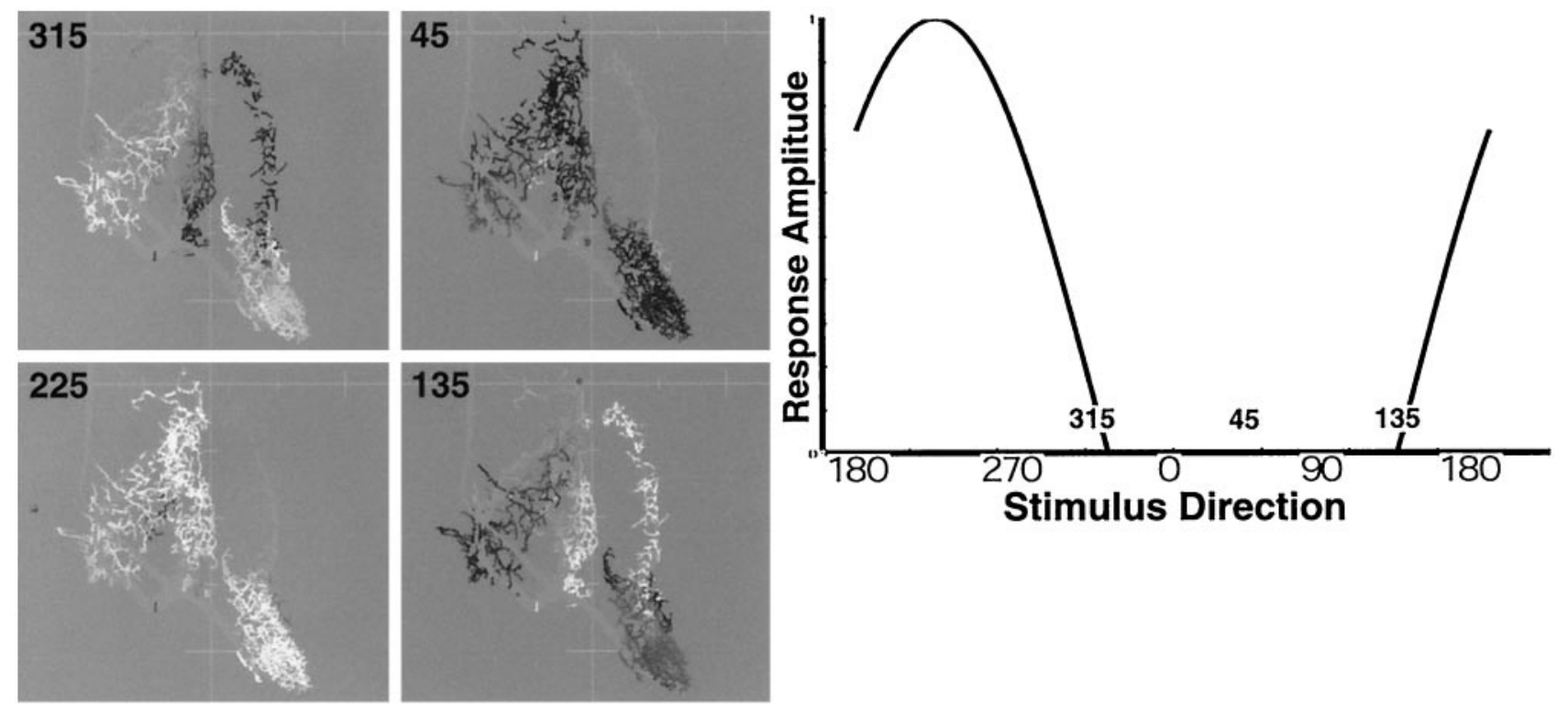

Figure 8. Prediction of the relative level of excitatory input to IN L10-2 in response to air currents from four orthogonal directions. The dendrites have been gray scale-coded according to the predicted level of excitatory input from the population of afferents, as in Figure 7. For each direction, the spatial pattern of activity elicited within the map is different, and thus the activation pattern masked onto the dendrites of the interneuron will appear different. The maximum level of activation occurs for $225^{\circ}$; the minimum level occurs for $45^{\circ}$. These directions correspond to the peak and trough, respectively, of the directional tuning curve of the cell. The response amplitude in the cell to directions 315 and $135^{\circ}$ is the same; however, the distribution of excitatory input to the cell is quite different for these two stimulus directions. The directional tuning curve of the cell is presented in right panel for reference. This tuning curve is a truncated cosine curve fit to physiologically measured tuning curves, as described in Materials and Methods.

that the arborizations from afferents with similar directional tuning properties are located near one another. Second, the afferent projection pattern is continuous with respect to stimulus direction: a continuous variation in stimulus direction causes a continuous variation in the activation pattern. Third, the restriction of the synaptic connections of an interneuron to a unique set of afferents having a limited range of directional sensitivities results from the unique anatomy of that interneuron: its dendritic arbors are located within restricted regions of the afferent map containing afferents with a limited subset of directional sensitivities.

Is this anatomically derived restriction to a subset of afferents sufficient to explain the directional tuning curves of the interneurons, or is it necessary to assume the operation of some additional constraints? To a first approximation, Figures 5 and 6 are consistent with the hypothesis that anatomical overlap between afferents and interneurons is an adequate indicator for their synaptic connectivity. That is, all essential features of the interneuron tuning curves can be understood under the assumptions that (1) each interneuron synapses with afferents that overlap with its dendritic arbors and (2) the relative synaptic weights of the connections between an interneuron and the different afferents are proportional to the relative overlap with those afferents.

We note that other interneurons in the cercal system show more complex directional response characteristics, including nonsinusoidal and bi-lobal directional tuning curves. Such directional tuning curves would not be predictable from linear summation of cosine basis functions. These complex response properties may emerge from various factors, including voltage-dependent conductances in the dendrites of the interneurons (Horner et al., 1997), complex properties of synaptic conductances and/or differential connectivity between interneurons, afferents of different length classes (Kanou and Shimozawa, 1984; Chiba et al., 1988;
Davis and Murphey, 1993), and synaptic interconnections with local interneurons.

\section{Functional significance of the structural organization of this system}

The functional organization of the set of four interneurons studied here is equivalent to a Cartesian coordinate system for computing the stimulus direction vector: the four interneurons project their cosine response sensitivity functions onto two perpendicular coordinate axes. For any air current stimulus direction, the firing rates of the two active interneurons could be decoded as Cartesian coordinates by neurons at successive processing stages. Four interneurons are required to cover all directions, because negative coordinates cannot be encoded within the region of the receptive field of a cell where its response is suppressed below firing threshold.

What is the functional significance of this Cartesian scheme? In an earlier study, we demonstrated that any variation in the spacing of the peaks of sensitivity of the four tuning curves away from perfect orthogonality would decrease encoding efficiency in this system, as measured by the mutual information between directional stimuli and the four-cell ensemble responses (Theunissen and Miller, 1991). Subsequently, Salinas and Abbott (1994) demonstrated that these four cells actually operate as an "optimal linear estimator" of stimulus direction. They showed that this Cartesian scheme is optimal in the sense that, on average, it provides the best possible linear reconstruction of the stimulus direction vector and works as well as more complex statistical methods that require more detailed information about the responses of the coding neurons.

Considering the functional significance of this arrangement, the developmental basis for the establishment of this Cartesian orthogonality of the directional sensitivity curves of these four 


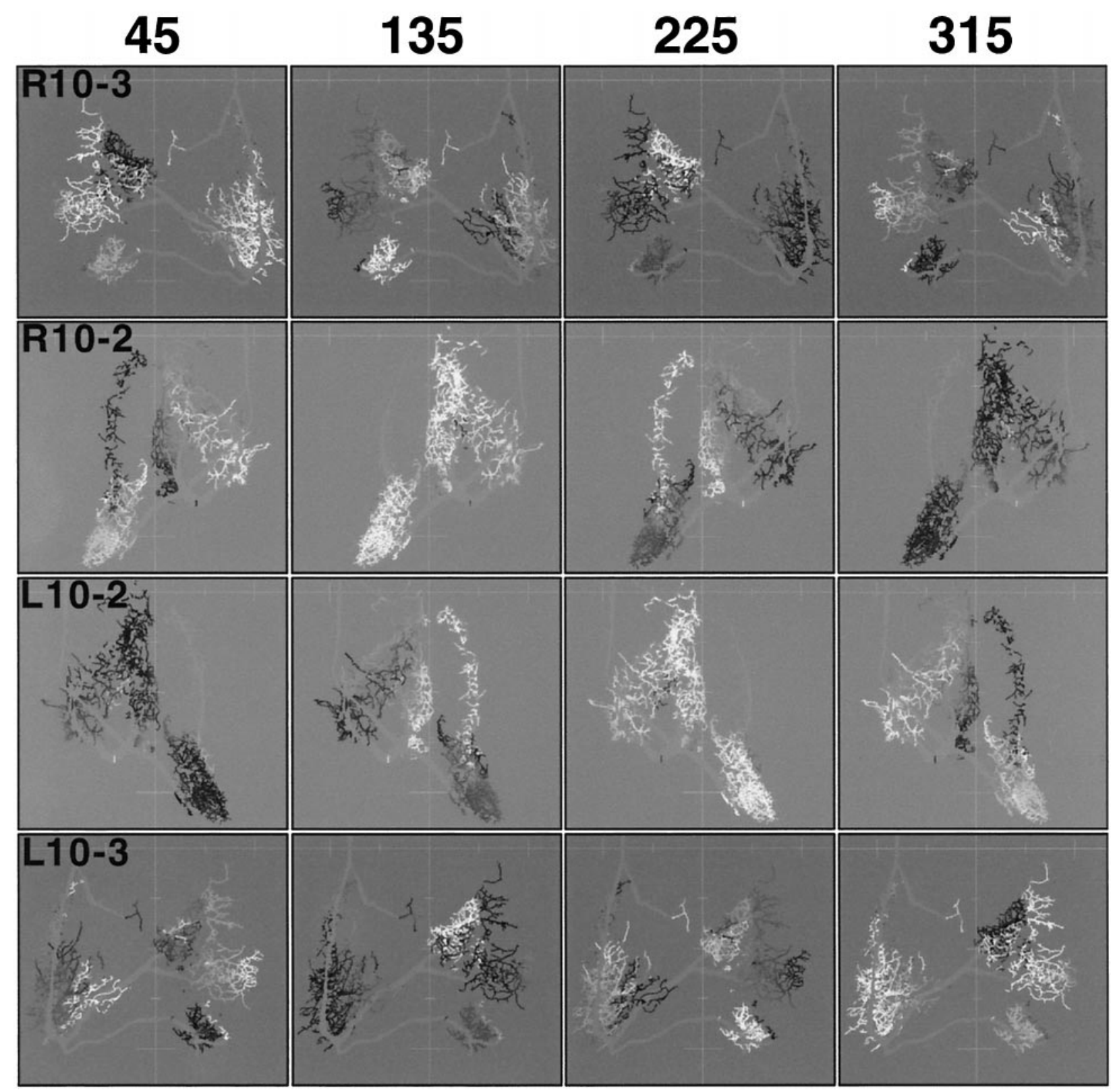

Figure 9. Predictions of the relative levels of excitatory input onto four interneurons in response to air currents from four orthogonal directions. Images were derived exactly as in Figures 7 and 8. Each panel of four images corresponds to the relative level of excitatory input to a single type of interneuron, in response to four different air current directions $\left(45,135,225\right.$, and $\left.315^{\circ}\right)$. For each direction, one interneuron receives the greatest excitatory input,

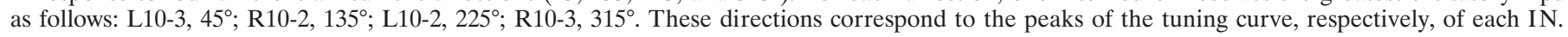

cells is extremely interesting. Certainly, this orthogonality is nontrivial: as shown in earlier work, the population of mechanosensory afferents on the cerci are not uniform with respect to the distribution of directional sensitivity curves (Landolfa and Jacobs, 1995). There are extreme peaks and valleys in the distribution of sensitivity curves, and the peaks are not coincident with the natural Cartesian axes established by the interneurons. Thus, for the interneurons to establish an orthogonal axis set, they may sample the set of afferent basis functions with a more complex synaptic weighting scheme that also meets the criteria of Salinas and Abbott (1994) for optimal linear estimation.

What factors might contribute to (or constrain) the establishment of this Cartesian system during development? To what extent is the connectivity scheme "hard-wired," and to what extent might it be activity dependent? On one hand, it seems reasonable to speculate that natural selection might have optimized a hard-wired, genetically preprogrammed configuration for this system that maximizes information processing capabilities under the constraints of limited resources. This system does, indeed, play a crucial role in defensive and reproductive behaviors. Alternatively, the orthogonal configuration of these four interneurons might be established and refined in an activitydependent manner during development. Although these two possibilities are impossible to distinguish on the basis of the data presented here, we note that the observed orthogonal configuration must correspond to a global minimum in the net covariance in activity across the set of four interneurons. This derives directly from the theoretical results of Salinas and Abbott (1994). 
To understand this at a more conceptual level, consider a case in which the tuning curve of one of the four interneurons was shifted "clockwise" with respect to the three other curves by $45^{\circ}$. The average covariance between its responses and those of its closest neighbor would increase significantly, and the degree of the negative covariance with its former polar opposite would decrease significantly. It is therefore conceivable that Hebbian-like activity-dependent plasticity mechanisms that follow correlation rules might play a role in fine-tuning the structural and/or functional parameters of this system.

The implementation of the coding scheme for the sensorimotor touch system in the leech is almost identical, in a functional sense, to the cercal system (Lewis and Kristan, 1998). Leeches respond to a touch on the body with a local bending reflex. The touch-sensitive $P$ neurons have cosine tuning curves that have peaks at 45, 135, 225, and $315^{\circ}$ with respect to the body axis, which are the same set of peak tuning angles of the four sensory interneurons in the cricket. Both of these systems are implemented around a Cartesian coordinate system, rotated with respect to the body axis by $45^{\circ}$.

Although the optimality of an orthogonal configuration can be derived from a functional standpoint, there are no known functional constraints that would favor the observed $45^{\circ}$ off-axis configurations. From the standpoint of our earlier informationtheoretic analysis or by the analysis of optimal linear estimators of Salinas and Abbott (1994), any set of four interneurons having appropriately shaped tuning curves should do as well as the actual set, as long as the peaks of the tuning curves of this alternate set are spaced at $90^{\circ}$ intervals around the sensory range. What additional constraints might "break the symmetry" in this case and select for the particular set of Cartesian coordinate axes seen in the cricket and leech systems? Here again, although mechanisms cannot be identified through our work, our results suggest one possibility: constraints might exist that are related to the establishment of bilateral symmetry of nervous systems. Each of the four interneurons that we studied has a homolog that is mirror symmetric across the ganglionic midplane. If we impose the constraint that any linear encoder must be constructed from bilaterally symmetric pairs of cells, then the observed $45^{\circ}$ configuration is the only truly optimal linear encoder, because it is the only configuration that can be constructed with only four cells. For any other orientation of the coordinate axes, a four-cell optimal linear estimator would have to include nonbilaterally symmetric cells. Alternatively, any other linear estimator that was constrained to use bilaterally symmetric cell pairs would require more than four (and therefore functionally redundant) neurons.

\section{REFERENCES}

Bacon JP, Altman JS (1977) A silver intensification method for cobalt filled neurons in whole mount preparations. Brain Res 138:359-363.

Bacon JP, Murphey RK (1984) Receptive fields of cricket (Acheta domesticus) are determined by their dendritic structure. J Physiol (Lond) 352:601-613.
Chiba A, Shepherd D, Murphey RK (1988) Synaptic rearrangement during postembryonic development in the cricket. Science 240:901-905.

Davis G, Murphey RK (1993) A role for postsynaptic neurons in determining presynaptic release properties in the cricket CNS: evidence for retrograde control of facilitation. J Neurosci 13:3827-3838.

Horner M, Kloppenburg P, Heblich R (1997) Characterization of ionic currents from identified cricket giant interneurons in primary cell culture. Proceedings of the 25th Gottingen Neurobiology Conference, Vol 2, p 780.

Jacobs GA, Miller JP (1985) Functional properties of individual neuronal branches isolated in situ by laser photoinactivation. Science 228:344-346.

Jacobs GA, Nevin R (1991) Anatomical relationships between sensory afferent arborizations in the cricket cercal system. Anat Rec 231:563-572.

Jacobs GA, Theunissen FE (1996) Functional organization of a neural map in the cricket cercal sensory system. J Neurosci 16:769-784.

Jacobs GA, Miller JP, Murphey RK (1986) Cellular mechanisms underlying directional sensitivity of an identified sensory interneuron. J Neurosci 6:2298-2311.

Johnson SE, Murphey RK (1985) The afferent projection of mesothoracic bristle hairs in the cricket Acheta domesticus. J Comp Physiol 156:369-379.

Kanou M, Shimozawa TA (1984) Threshold analysis of cricket cercal interneurons by an alternating air-current stimulus. J Comp Physiol [A] 154:357-365.

Landolfa M, Jacobs GA (1995) Direction sensitivity of the filiform hair population of the cricket cercal system. J Comp Physiol [A] 177:759-766

Landolfa MA, Miller JP (1995) Stimulus/response properties of cricket cercal filiform hair receptors. J Comp Physiol [A] 177:749-757.

Lewis JE, Kristan WB (1998) A neuronal network of computing population vectors in the leech. Nature 391:76-79.

Miller JP, Jacobs GA, Theunissen FE (1991) Representation of sensory information in the cricket cercal sensory system. I. Response properties of the primary interneurons. J Neurophysiol 66:1680-1689.

Paydar S, Doan CA, Jacobs GA (1999) Neural mapping of direction and frequency in the cricket cercal sensory system. J Neurosci 19:1771-1781.

Roddey JC, Jacobs GA (1996) Information theoretic analysis of dynamical encoding by filiform mechanoreceptors in the cricket cercal system J Neurophysiol 75:1365-1476.

Salinas E, Abbott LF (1994) Vector reconstruction from firing rates. J Comput Neurosci 1:89-107.

Shimozawa T, Kanou M (1984a) Varieties of filiform hairs: range fractionation by sensory afferents and cercal interneurons of a cricket. J Comp Physiol [A] 155:485-493.

Shimozawa T, Kanou M (1984b) The aerodynamics and sensory physiology of range fractionation in the cercal filiform sensilla of the cricket Gryllus bimaculatus. J Comp Physiol [A] 155:495-505.

Theunissen FE, Miller JP (1991) Representation of sensory information in the cricket cercal sensory system. II. Information theoretic calculation of system accuracy and optimal tuning-curve widths of four primary interneurons. J Neurophysiol 66:1690-1703.

Theunissen FE, Roddey JC, Stufflebeam S, Clague H, Miller JP (1996) Information theoretic analysis of dynamical encoding by four primary sensory interneurons in the cricket cercal system. J Neurophysiol 75:1345-1376.

Troyer TW, Levin JE, Jacobs GA (1994) Construction and analysis of a data base representing a neural map. Microsc Res Tech 29:329-343. 